\title{
Free Convection Flow between Vertical Plates Moving in Opposite Direction and Partially Filled with Porous Medium
}

\author{
Umesh Gupta ${ }^{1}$, Abhay Kumar Jha ${ }^{2}$, Rama Charan Chaudhary ${ }^{3}$ \\ ${ }^{1}$ Department of Mathematics, Institute of Engineering and Technology, JK Lakshmipat University, Jaipur, India \\ ${ }^{2}$ Department of Mathematics, Apex Institute of Engineering and Technology, Jaipur, India \\ ${ }^{3}$ Yagvalkya Institute of Technology, Jaipur, India \\ E-mail: umeshindian@yahoo.com,itsabhay@rediffmail.com,rcchaudhary@rediffmail.com \\ Received January 25, 2011; revised June 18, 2011; accepted June 25, 2011
}

\begin{abstract}
The laminar fully developed free-convection flow in a channel bounded by two vertical plates, partially filled with porous matrix and partially with a clear fluid, has been discussed when both the plates are moving in opposite direction. The momentum transfer in porous medium has been described by the Brinkman-extended Darcy model. The affect of Darcy number on flow velocity has been discussed in fluid region, interface region and porous medium with the help of graphs. Analytic method has been adopted to obtain the expressions of velocity and temperature. The skin-friction component has also been determined and presented with the help of tables.
\end{abstract}

Keywords: Free Convection, Porous Medium, Skin-Friction

\section{Introduction}

Flow in a region, part of which is occupied by a clear fluid and part by a fluid-saturated porous medium, has recently attracted considerable attention due to its common occurrence in both geophysical and industrial environments, including engineering applications such as thermal-energy storage system, a solar collector with a porous absorber and porous journal-bearings. Flow mechanism at the fluid/porous interface, was first studied by Beavers \& Joseph [1] and it was investigated that the velocity gradient on the fluid side of the interface is proportional to the slip velocity at the interface. Taylor [2] \& Richardson [3] continued the investigation in which they modeled fluid flow by Darcy number. Further, Vafai \& Kim [4] modeled the flow in the porous region utilizing the so-called Brinkman-Forchheimer-extended Darcy equation (Vafai \& Kim [5] and Kuznetsov [6]). Alazmi \& Vafai [7], Valencia-Lopez and Ochoa-Tapia [8] also investigated fluid flow and heat transfer interfacial conditions of fluid and porous layers.

Furthermore, convection in porous media is applied in utilization of geothermal energy, the control of pollutant spread in groundwater, the design of nuclear reactors, compact heat exchangers, solar power collectors, heat transfer associated with the deep storage of nuclear waste and high performance insulators for buildings. Considerable progress in this area was made by Nield \& Bejan [9] and Kaviany [10]. Vafai \& Tien [11] also analyzed the effects of a solid boundary and the inertial forces on flow and heat transfer in porous media.

The coupled fluid flow and heat transfer problem in a fully developed composite region of two parallel plates filled with Brinkman-Darcy porous medium was analytically investigated by Kaviany [12]. Rudraiah \& Nagraj [13] studied the fully developed free-convection flow of a viscous fluid through a porous medium bounded by two heated vertical plates. Beckerman [14] studied natural convection in vertical enclosures containing simultaneously fluid and porous layers. Recently, Khalili et al. [15] studied the instability of superimposed fluid and porous layers with vertical through-flow governed by Darcy-Forchheimer equation. Free convection between vertical walls partially filled with porous medium was investigated by Paul et al. [16]. Singh et al. [17] analyzed heat and mass transfer phenomena due to 
natural convection in a composite cavity containing a fluid layer overlying a porous layer saturated with the same fluid, in which the flow in the porous region was modeled using Brinkman-Forchheimer-extended Darcy model that includes both the effect of macroscopic shear (Brinkman effect) and flow inertia (Forchheimer effect).

In most of the flow studies in the channel, the plates are stable. However, to the best of the author's knowledge, the effect of the same idea has not been studied yet, when both the plates are moving. In this paper we extend the problem of [16], when both the plates are moving in the opposite direction.

\section{Governing Equations}

A channel of two vertical plates partially filled with porous matrix and partially with a clear fluid having interface is shown in Figure 1. The laminar fully developed free-convection flow bounded in the channel is discussed, when both the plates are moving in opposite direction and one plate is heated and other is cooled.

The $\bar{x}$-axis is taken along one of the wall and $\bar{y}$ axis normal to it. The plate in the fluid region and the plate in porous region are moving in opposite direction, where $\bar{U}_{f}$ and $\bar{U}_{p}$ are the velocities in the direction of $\bar{x}$-axis. The temperature is also considered on the walls $\bar{y}=0$ and $\bar{y}=H$ as $\bar{T}_{f}=\bar{T}_{c}+A\left(\bar{T}_{h}-\bar{T}_{c}\right)$ and $\bar{T}_{p}=\bar{T}_{c}+B\left(\bar{T}_{h}-\bar{T}_{c}\right)$ respectively.

Under usual Boussinesq approximation, the flow in fluid and porous regions is governed by the following equations:

Free Fluid Region:

$$
\frac{\mathrm{d}^{2} \bar{U}_{f}}{\mathrm{~d} \bar{y}^{2}}+\frac{g \beta}{v}\left(\bar{T}_{f}-\bar{T}_{c}\right)=0
$$

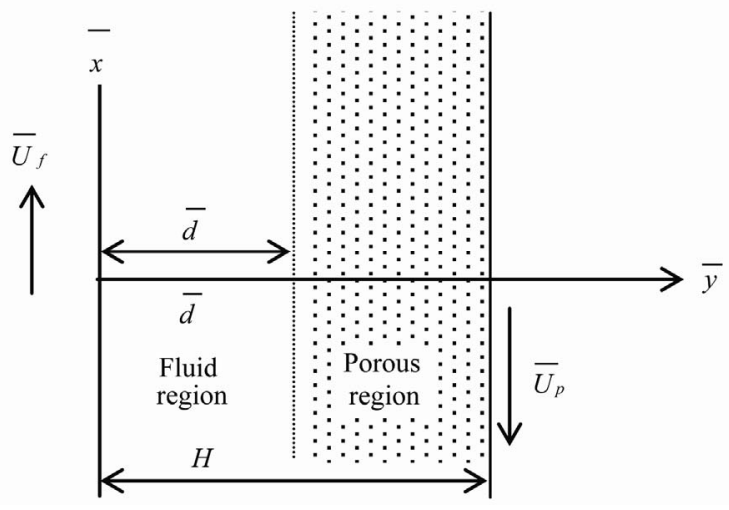

Figure 1. Physical configuration of the system.

$$
\left(\frac{\mathrm{d} \bar{U}_{f}}{\mathrm{~d} \bar{y}}\right)^{2}+\frac{\kappa}{v} \frac{\mathrm{d}^{2}\left(\bar{T}_{f}-\bar{T}_{c}\right)}{\mathrm{d} \bar{y}^{2}}=0
$$

Porous Region:

$$
\begin{gathered}
\frac{\mathrm{d}^{2} \bar{U}_{p}}{\mathrm{~d} \bar{y}^{2}}-\frac{\bar{U}_{p}}{\bar{k}}+\frac{g \beta}{v}\left(\bar{T}_{p}-\bar{T}_{c}\right)=0 \\
\left(\frac{\mathrm{d} \bar{U}_{p}}{\mathrm{~d} \bar{y}}\right)^{2}+\frac{\bar{U}_{p}^{2}}{\bar{k}}+\frac{\kappa}{v} \frac{\mathrm{d}^{2}\left(\bar{T}_{p}-\bar{T}_{c}\right)}{\mathrm{d} \bar{y}^{2}}=0
\end{gathered}
$$

The corresponding boundary conditions are

$$
\left.\begin{array}{l}
\bar{y}=0: \bar{U}_{f}=\frac{g \beta H^{2}\left(\bar{T}_{h}-\bar{T}_{c}\right) u_{0}}{v} ; \bar{T}_{f}=\bar{T}_{c}+A\left(\bar{T}_{h}-\bar{T}_{c}\right) \\
\bar{y}=H: \bar{U}_{p}=-\frac{g \beta H^{2}\left(\bar{T}_{h}-\bar{T}_{c}\right) u_{0}}{v} ; \bar{T}_{p}=\bar{T}_{c}+B\left(\bar{T}_{h}-\bar{T}_{c}\right) \\
\bar{y}=\bar{d}: \bar{U}_{f}=\bar{U}_{p} ; \frac{\mathrm{d} \bar{U}_{f}}{\mathrm{~d} \bar{y}}=\frac{\mathrm{d} \bar{U}_{p}}{\mathrm{~d} \bar{y}} ; \bar{T}_{f}=\bar{T}_{p} ; \frac{\mathrm{d} \bar{T}_{f}}{\mathrm{~d} \bar{y}}=\frac{\mathrm{d} \bar{T}_{p}}{\mathrm{~d} \bar{y}}
\end{array}\right\}
$$

Introducing following non-dimensional quantities:

$$
\begin{aligned}
& D a=\frac{\bar{k}}{H^{2}} ; \quad y=\frac{\bar{y}}{H} ; d=\frac{\bar{d}}{H} ; U_{f}=\frac{v \bar{U}_{f}}{g \beta H^{2}\left(\bar{T}_{h}-\bar{T}_{c}\right)} ; \\
& U_{p}=\frac{v \bar{U}_{p}}{g \beta H^{2}\left(\bar{T}_{h}-\bar{T}_{c}\right)} ; \theta_{f}=\frac{\left(\bar{T}_{f}-\bar{T}_{c}\right)}{\left(\bar{T}_{h}-\bar{T}_{c}\right)} ; \\
& \theta_{p}=\frac{\left(\bar{T}_{p}-\bar{T}_{c}\right)}{\left(\bar{T}_{h}-\bar{T}_{c}\right)} ; \quad N=\frac{g^{2} \beta^{2} H^{4}\left(\bar{T}_{h}-\bar{T}_{c}\right)}{\kappa v}
\end{aligned}
$$

Equations (1) to (4) become

Fluid Region:

$$
\begin{gathered}
\frac{\mathrm{d}^{2} U_{f}}{\mathrm{~d} y^{2}}+\theta_{f}=0 \\
\frac{\mathrm{d}^{2} \theta_{f}}{\mathrm{~d} y^{2}}+N\left(\frac{\mathrm{d} U_{f}}{\mathrm{~d} y}\right)^{2}=0
\end{gathered}
$$

Porous Region:

$$
\begin{gathered}
\frac{\mathrm{d}^{2} U_{p}}{\mathrm{~d} y^{2}}-\frac{U_{p}}{D a}+\theta_{p}=0 \\
\frac{\mathrm{d}^{2} \theta_{p}}{\mathrm{~d} y^{2}}+N\left(\frac{\mathrm{d} U_{p}}{\mathrm{~d} y}\right)^{2}+\frac{N}{D a} U_{p}^{2}=0
\end{gathered}
$$


In Equation (9), The momentum transfer in porous domain is described based on Brinkman-extended Darcy model [18].

Where Da is the Darcy number, $\mathrm{d}$ the distance of interface from the plate $y=0, g$ the acceleration due to gravity, $H$ the distance between vertical plates, $\bar{k}$ the permeability of the porous matrix, $\kappa$ the thermal conductivity, $\mathrm{N}$ the Buoyancy parameter, $\beta$ the coefficient of thermal expansion, $\mu$ the dynamic viscosity, $v$ the kinematic viscosity, $\rho$ the density, and $\theta$ is the temperature. The subscripts $f$, represent fluid layer, $p$ porous layer, $h$ hot plate and $c$, the cold plate.

The boundary and matching conditions (5) in dimensionless form are:

$$
\begin{aligned}
& y=0: U_{f}=u_{0} ; \theta_{f}=A \\
& y=1: U_{p}=-u_{0} ; \theta_{f}=B \\
& y=d: U_{f}=U_{p} ; \frac{\mathrm{d} U_{f}}{\mathrm{~d} y}=\frac{\mathrm{d} U_{p}}{\mathrm{~d} y} \\
& y=d: \theta_{f}=\theta_{p} ; \frac{\mathrm{d} \theta_{f}}{\mathrm{~d} y}=\frac{\mathrm{d} \theta_{p}}{\mathrm{~d} y}
\end{aligned}
$$

where, the matching conditions for velocity are due to continuity of velocity and shear stress at the interface. The continuity of temperature and heat flux at the interface has been considered as matching conditions for temperature.

\section{Solution}

It can be observed that problem is non-linear due to viscous and Darcy dissipation terms. This problem can be tackled by using a perturbation method as $\mathrm{N}$ is small in most of the practical problems. Accordingly we assume, for small $\mathrm{N}$, the expansions:

$$
\begin{aligned}
& U_{f}=U_{0 f}+N U_{1 f}+O\left(N^{2}\right) \\
& U_{p}=U_{0 p}+N U_{1 p}+O\left(N^{2}\right) \\
& \theta_{f}=\theta_{0 f}+N \theta_{1 f}+O\left(N^{2}\right) \\
& \theta_{p}=\theta_{0 p}+N \theta_{1 p}+O\left(N^{2}\right)
\end{aligned}
$$

Substituting (12) in the Equations (7) to (10) gives

$$
\begin{aligned}
& \frac{\mathrm{d}^{2} U_{0 f}}{\mathrm{~d} y^{2}}+\theta_{0 f}=0 \\
& \frac{\mathrm{d}^{2} U_{1 f}}{\mathrm{~d} y^{2}}+\theta_{1 f}=0 \\
& \frac{\mathrm{d}^{2} \theta_{0 f}}{\mathrm{~d} y^{2}}=0
\end{aligned}
$$

$$
\begin{gathered}
\frac{\mathrm{d}^{2} \theta_{1 f}}{\mathrm{~d} y^{2}}+\left(\frac{\mathrm{d} U_{0 f}}{\mathrm{~d} y}\right)^{2}=0 \\
\frac{\mathrm{d}^{2} U_{0 p}}{\mathrm{~d} y^{2}}-\frac{1}{D a} U_{0 p}+\theta_{0 p}=0 \\
\frac{\mathrm{d}^{2} U_{1 p}}{\mathrm{~d} y^{2}}-\frac{1}{D a} U_{1 p}+\theta_{1 p}=0 \\
\frac{\mathrm{d}^{2} \theta_{0 p}}{\mathrm{~d} y^{2}}=0 \\
\frac{\mathrm{d}^{2} \theta_{1 p}}{\mathrm{~d} y^{2}}+\left(\frac{\mathrm{d} U_{0 p}}{\mathrm{~d} y}\right)^{2}+\frac{1}{D a} U_{0 p}^{2}=0
\end{gathered}
$$

The corresponding boundary conditions are

$$
\begin{aligned}
& y=0: \quad U_{0 f}=u_{0} ; U_{1 f}=0 ; \theta_{0 f}=A ; \theta_{1 f}=0 ; \\
& y=1: \quad U_{0 p}=u_{0} ; U_{1 p}=0 ; \theta_{0 p}=B ; \theta_{1 p}=0 ; \\
& y=d: \\
& \quad U_{0 f}=U_{0 p} ; U_{1 f}=U_{1 p} ; \frac{\mathrm{d} U_{0 f}}{\mathrm{~d} y}=\frac{\mathrm{d} U_{0 p}}{\mathrm{~d} y} ; \frac{\mathrm{d} U_{1 f}}{\mathrm{~d} y}=\frac{\mathrm{d} U_{1 p}}{\mathrm{~d} y} \\
& \quad \theta_{0 f}=\theta_{0 p} ; \theta_{1 f}=\theta_{1 p} ; \frac{\mathrm{d} \theta_{0 f}}{\mathrm{~d} y}=\frac{\mathrm{d} \theta_{0 p}}{\mathrm{~d} y} ; \frac{\mathrm{d} \theta_{1 f}}{\mathrm{~d} y}=\frac{\mathrm{d} \theta_{1 p}}{\mathrm{~d} y}
\end{aligned}
$$

Solving Equations (13) to (20) using boundary conditions (21) gives the following velocity and temperature components

$$
\begin{aligned}
U_{0 f} & =-\frac{A y^{2}}{2}+(A-B) \frac{y^{3}}{6}+u_{0}+K_{5} y \\
U_{0 p} & =\frac{A+(B-A) y}{m^{2}}+K_{4} \mathrm{e}^{m y}+K_{3} \mathrm{e}^{-m y} \\
\theta_{0 f} & =\theta_{0 p}=A+(B-A) y \\
\theta_{1 f} & =-\frac{(A-B)^{2} y^{6}}{120}+\frac{A(A-B) y^{5}}{20}-\frac{K_{5}(A-B) y^{4}}{12} \\
& -\frac{A^{2} y^{4}}{12}+\frac{A K_{5} y^{3}}{3}-\frac{K_{5}^{2} y^{2}}{2}+K_{13} y \\
\theta_{1 p}= & -\frac{K_{4}^{2} \mathrm{e}^{2 m y}}{2}-\frac{K_{3}^{2} \mathrm{e}^{-2 m y}}{2}-\frac{A^{2} y^{2}}{2 m^{2}}-\frac{(B-A)^{2} y^{2}}{2 m^{4}} \\
- & \frac{(B-A)^{2} y^{4}}{12 m^{2}}-\frac{A(B-A) y^{3}}{3 m^{2}}-\frac{2 K_{4} \mathrm{e}^{m y}}{m^{2}}\left(A-\frac{B-A}{m}\right) \\
- & \frac{2 K_{3} \mathrm{e}^{-m y}}{m^{2}}\left(A+\frac{B-A}{m}\right)-\frac{2 K_{4}(B-A) y \mathrm{e}^{m y}}{m^{2}} \\
- & \frac{2 K_{3}(B-A) y \mathrm{e}^{-m y}}{m^{2}}+K_{12} y+K_{11}
\end{aligned}
$$




$$
\begin{aligned}
U_{1 f} & =\frac{(A-B)^{2} y^{8}}{6720}-\frac{A(A-B) y^{7}}{840}+\frac{K_{5}(A-B) y^{6}}{360} \\
& +\frac{A^{2} y^{6}}{360}-\frac{A K_{5} y^{5}}{60}+\frac{K_{5}^{2} y^{4}}{24}-\frac{K_{13} y^{3}}{6}+K_{22} y \\
U_{1 p} & =K_{21} \mathrm{e}^{m y}+K_{20} \mathrm{e}^{-m y}+\frac{K_{4}^{2} \mathrm{e}^{2 m y}}{6 m^{2}}+\frac{K_{3}^{2} \mathrm{e}^{-2 m y}}{6 m^{2}} \\
& -\frac{1}{2 m^{6}}\left(m^{2} y^{2}+2\right)\left(A^{2}+\frac{(A-B)^{2}}{m^{2}}\right)-\frac{(B-A)^{2}}{12 m^{8}} \\
& \cdot\left(m^{4} y^{4}+12 m^{2} y^{2}+24\right)+\frac{K_{4}}{2 m^{4}}\left(A-\frac{(B-A)}{m}\right) \\
& \cdot \mathrm{e}^{m y}(2 m y-1)-\frac{A(B-A) y}{3 m^{6}}\left(m^{2} y^{2}+6\right) \\
& -\frac{K_{3}}{2 m^{4}}\left(A+\frac{(B-A)}{m}\right) \mathrm{e}^{-m y}(2 m y+1)+\frac{K_{4}(B-A) e^{m y}}{4 m^{5}} \\
& \cdot\left(2 m^{2} y^{2}-2 m y+1\right)-\frac{K_{3}(B-A) \mathrm{e}^{-m y}}{4 m^{5}} \\
& \cdot\left(2 m^{2} y^{2}+2 m y+1\right)+\frac{K_{12} y+K_{11}}{m^{2}}
\end{aligned}
$$

where

$$
m=(D a)^{-1 / 2} ;
$$$$
K_{1}=\frac{A d^{2}}{2}-\frac{(A-B) d^{3}}{3}+u_{0} ;
$$$$
K_{2}=(1+m d) \mathrm{e}^{m(1-d)}-(1-m d) \mathrm{e}^{-m(1-d)} ;
$$$$
K_{3}=\frac{1}{K_{2}}\left[\mathrm{e}^{m}\left(K_{1}-\frac{A}{m^{2}}\right)+(1-m d) \mathrm{e}^{m d}\left(\frac{B}{m^{2}}+u_{0}\right)\right] ;
$$$$
K_{4}=\left(-u_{0}-\frac{B}{m^{2}}\right) \mathrm{e}^{-m}-K_{3} \mathrm{e}^{-2 m} \text {; }
$$$$
K_{5}=\frac{1}{d}\left[K_{4} \mathrm{e}^{m d}+K_{3} \mathrm{e}^{-m d}+\frac{A+(B-A) d}{m^{2}}\right.
$$$$
\left.+\frac{A d^{2}}{2}-\frac{(A-B) d^{3}}{6}-u_{0}\right]
$$$$
K_{6}=-\frac{(A-B)^{2} d^{5}}{20}+\frac{A(A-B) d^{4}}{4}-\frac{A^{2} d^{3}}{3}
$$$$
-\frac{K_{5}(A-B) d^{3}}{3}+A K_{5} d^{2}-K_{5}^{2} d ;
$$

$$
\begin{aligned}
K_{7}= & -\frac{(A-B)^{2} d^{6}}{120}+\frac{A(A-B) d^{5}}{20}-\frac{A^{2} d^{4}}{12} \\
& -\frac{K_{5}(A-B) d^{4}}{12}+\frac{A K_{5} d^{3}}{3}-\frac{K_{5}^{2} d^{2}}{2} ;
\end{aligned}
$$

$$
\begin{aligned}
K_{8}= & -\frac{K_{4}^{2} \mathrm{e}^{2 m}}{2}-\frac{K_{3}^{2} \mathrm{e}^{-2 m}}{2}-\frac{A^{2}}{2 m^{2}}-\frac{(B-A)^{2}}{2 m^{4}} \\
& -\frac{(B-A)^{2}}{12 m^{2}}-\frac{A(B-A)}{3 m^{2}}-\frac{2 K_{4} \mathrm{e}^{m}}{m^{2}}\left(A-\frac{B-A}{m}\right) \\
& -\frac{2 K_{3} \mathrm{e}^{-m}}{m^{2}}\left(A+\frac{B-A}{m}\right)-\frac{2 K_{4}(B-A) e^{m}}{m^{2}} \\
& -\frac{2 K_{3}(B-A) \mathrm{e}^{-m}}{m^{2}} ; \\
K_{9} & =-\frac{K_{4}^{2} \mathrm{e}^{2 m d}}{2}-\frac{K_{3}^{2} \mathrm{e}^{-2 m d}}{2}-\frac{A^{2} d^{2}}{2 m^{2}} \\
& -\frac{(B-A)^{2} d^{2}}{2 m^{4}}-\frac{(B-A)^{2} d^{4}}{12 m^{2}}-\frac{A(B-A) d^{3}}{3 m^{2}} \\
& -\frac{2 K_{4} \mathrm{e}^{m d}}{m^{2}}\left(A-\frac{B-A}{m}\right)-\frac{2 K_{3} \mathrm{e}^{-m d}}{m^{2}}\left(A+\frac{B-A}{m}\right) \\
& -\frac{2 K_{4}(B-A) d \mathrm{e}^{m d}}{m^{2}}-\frac{2 K_{3}(B-A) d \mathrm{e}^{-m d}}{m^{2}} ;
\end{aligned}
$$$$
K_{10}=-m K_{4}^{2} \mathrm{e}^{2 m d}+m K_{3}^{2} \mathrm{e}^{-2 m d}-\frac{A^{2} d}{m^{2}}-\frac{(B-A)^{2} d}{m^{4}}
$$$$
-\frac{(B-A)^{2} d^{3}}{3 m^{2}}-\frac{A(B-A) d^{2}}{m^{2}}-\frac{2 A K_{4} \mathrm{e}^{m d}}{m}
$$$$
+\frac{2 A K_{3} \mathrm{e}^{-m d}}{m}-\frac{2 K_{4}(B-A) d \mathrm{e}^{m d}}{m}
$$$$
+\frac{2 K_{3}(B-A) d \mathrm{e}^{-m d}}{m} ;
$$$$
K_{11}=\left(K_{10}-K_{6}\right) d-K_{9}+K_{7} ;
$$$$
K_{12}=-K_{8}-K_{11} \text {; }
$$$$
K_{13}=K_{10}-K_{6}+K_{12} \text {; }
$$$$
K_{14}=\frac{K_{4}^{2} \mathrm{e}^{2 m}}{6 m^{2}}+\frac{K_{3}^{2} \mathrm{e}^{-2 m}}{6 m^{2}}-\frac{1}{2 m^{6}}\left(m^{2}+2\right)\left(A^{2}+\frac{(A-B)^{2}}{m^{2}}\right)
$$$$
-\frac{(A-B)^{2}}{12 m^{8}}\left(m^{4}+12 m^{2}+24\right)+\frac{K_{4}}{2 m^{4}}\left(A-\frac{(B-A)}{m}\right)
$$$$
\cdot \mathrm{e}^{m}(2 m-1)-\frac{A(B-A)}{3 m^{6}}\left(m^{2}+6\right)-\frac{K_{3}}{2 m^{4}}
$$$$
\cdot\left(A+\frac{(B-A)}{m}\right) \mathrm{e}^{-m y}(2 m+1)+\frac{K_{4}(B-A)}{4 m^{5}}
$$$$
\cdot\left(2 m^{2}-2 m+1\right)-\frac{K_{3}(B-A)}{4 m^{5}}\left(2 m^{2}+2 m+1\right)
$$$$
+\frac{K_{12}+K_{11}}{m^{2}} \text {; }
$$ 


$$
\begin{aligned}
& K_{15}=\frac{(A-B)^{2} d^{8}}{6720}-\frac{A(A-B) d^{7}}{840} \\
& +\frac{K_{5}(A-B) d^{6}}{360}+\frac{A^{2} d^{6}}{360}-\frac{A K_{5} d^{5}}{60} \\
& +\frac{K_{5}^{2} d^{4}}{24}-\frac{K_{13} d^{3}}{6} \text {; } \\
& K_{16}=\frac{K_{4}^{2} \mathrm{e}^{2 m d}}{6 m^{2}}+\frac{K_{3}^{2} \mathrm{e}^{-2 m d}}{6 m^{2}}-\frac{1}{2 m^{6}}\left(m^{2} d^{2}+2\right) \\
& \left(A^{2}+\frac{(A-B)^{2}}{m^{2}}\right)-\frac{(B-A)^{2}}{12 m^{8}}\left(m^{4} d^{4}+12 m^{2} d^{2}+24\right) \\
& +\frac{K_{4}}{2 m^{4}}\left(A-\frac{(B-A)}{m}\right) \mathrm{e}^{m d}(2 m d-1)-\frac{A(B-A) d}{3 m^{6}} \\
& \cdot\left(m^{2} d^{2}+6\right)-\frac{K_{3}}{2 m^{4}}\left(A+\frac{(B-A)}{m}\right) \mathrm{e}^{-m d}(2 m d+1) \\
& +\frac{K_{4}(B-A) \mathrm{e}^{m d}}{4 m^{5}}\left(2 m^{2} d^{2}-2 m d+1\right)-\frac{K_{3}(B-A) \mathrm{e}^{-m d}}{4 m^{5}} \\
& \cdot\left(2 m^{2} d^{2}+2 m d+1\right)+\frac{K_{12} d+K_{11}}{m^{2}} \\
& K_{17}=\frac{(A-B)^{2} d^{7}}{840}-\frac{A(A-B) d^{6}}{120}+\frac{K_{5}(A-B) d^{5}}{60} \\
& +\frac{A^{2} d^{5}}{60}-\frac{A K_{5} d^{4}}{12}+\frac{K_{5}^{2} d^{3}}{6}-\frac{K_{13} d^{2}}{2} ; \\
& K_{18}=\frac{K_{4}^{2} \mathrm{e}^{2 m d}}{3 m}-\frac{K_{3}^{2} \mathrm{e}^{-2 m d}}{3 m}-\frac{d}{m^{4}}\left(A^{2}+\frac{(A-B)^{2}}{m^{2}}\right) \\
& -\frac{(B-A)^{2}}{3 m^{6}} d\left(m^{2} d^{2}+6\right)-\frac{A(B-A)}{m^{6}}\left(m^{2} d^{2}+2\right) \\
& +\frac{K_{4}}{2 m^{3}}\left(A-\frac{(B-A)}{m}\right) \mathrm{e}^{m d}(2 m d+1)-\frac{K_{3}}{2 m^{3}} \\
& \cdot\left(A+\frac{(B-A)}{m}\right) \mathrm{e}^{-m d}(1-2 m d)+\frac{K_{4}(B-A)}{2 m^{4}} \\
& \cdot \mathrm{e}^{m d}(2 m d-1)+\frac{K_{4}(B-A) \mathrm{e}^{m d}}{4 m^{4}}\left(2 m^{2} d^{2}-2 m d+1\right) \\
& +\frac{K_{3}(B-A) \mathrm{e}^{-m d}}{4 m^{4}}\left(2 m^{2} d^{2}+2 m d+1\right) \\
& -\frac{K_{3}(B-A) \mathrm{e}^{-m d}}{2 m^{4}}(2 m d+1)+\frac{K_{12}}{m^{2}} ; \\
& K_{19}=(1-m d) K_{14} \mathrm{e}^{m d}-\left[\left(K_{16}-K_{15}\right)-d\left(K_{18}-K_{17}\right)\right] \mathrm{e}^{m} ; \\
& K_{20}=K_{19} / K_{2} \text {; }
\end{aligned}
$$

$$
\begin{aligned}
& K_{21}=-K_{14} \mathrm{e}^{-m}-K_{20} \mathrm{e}^{-2 m} ; \\
& K_{22}=m K_{21} \mathrm{e}^{m d}-m K_{20} \mathrm{e}^{-m d}+K_{18}-K_{17} .
\end{aligned}
$$

Skin-Friction Components

At plate $y=0$ :

$$
\tau_{1}=K_{5}+N K_{22}
$$

At plate $y=1$ :

$$
\begin{aligned}
\tau_{2} & =-\left[\frac{(B-A)}{m^{2}}+m K_{4} \mathrm{e}^{m}-m K_{3} \mathrm{e}^{-m}\right] \\
& -N\left[\frac{K_{4}^{2} \mathrm{e}^{2 m}}{3 m}-\frac{K_{3}^{2} \mathrm{e}^{-2 m}}{3 m}-\frac{1}{m^{4}}\left(A^{2}+\frac{(A-B)^{2}}{m^{2}}\right)\right. \\
& -\frac{(B-A)^{2}}{3 m^{6}}\left(m^{2}+6\right)-\frac{A(B-A)}{m^{6}}\left(m^{2}+2\right)+\frac{K_{4}}{2 m^{3}} \\
& \cdot\left(A-\frac{(B-A)}{m}\right) \mathrm{e}^{m}(2 m+1)-\frac{K_{3}}{2 m^{3}}\left(A+\frac{(B-A)}{m}\right) \\
& \cdot \mathrm{e}^{-m}(1-2 m)+\frac{K_{4}(B-A)}{2 m^{4}} \mathrm{e}^{m}(2 m-1)+\frac{K_{4}(B-A) \mathrm{e}^{m}}{4 m^{4}} \\
& \cdot\left(2 m^{2}-2 m+1\right)+\frac{K_{3}(B-A) \mathrm{e}^{-m}}{4 m^{4}}\left(2 m^{2}+2 m+1\right) \\
& \left.-\frac{K_{3}(B-A) \mathrm{e}^{-m}}{2 m^{4}}(2 m+1)+\frac{K_{12}}{m^{2}}+m K_{21} \mathrm{e}^{m}-m K_{20} \mathrm{e}^{-m}\right]
\end{aligned}
$$

\section{Discussions and Conclusion}

Natural convection in a vertical channel partially filled with porous medium has been discussed in the preceding sections when it is assumed that plates are moving in opposite direction. The governing equations having nonlinear nature have been solved by analytical method. Different types of interfacial conditions between a porous medium and fluid layer are analyzed in detail. Three primary regions were found likewise, fluid region (near wall $y=0$ ), interface region and porous region (near the wall $y=1$ ). The affect of Darcy number on the flow has been discussed.

When viscous and Darcy dissipation are zero, velocity profiles between vertical plates have been illustrated in Figure 2, for the cases when $A=1, B=1$ (plate $y=0$ is heated and plate $y=1$ is cooled) and $A=0, B=1$ (plate $y=0$ is cooled and $y=1$ is heated). It is observed that in first region, the fluid velocity increases with increasing Darcy number (Da), whereas the reversal phenomenon occurs in third region. Near the interface region in porous medium, the velocity is constant for Da $=10^{-3}$ and effect of Brinkman term is almost negligible, and flow is only characterized by classical Darcy 


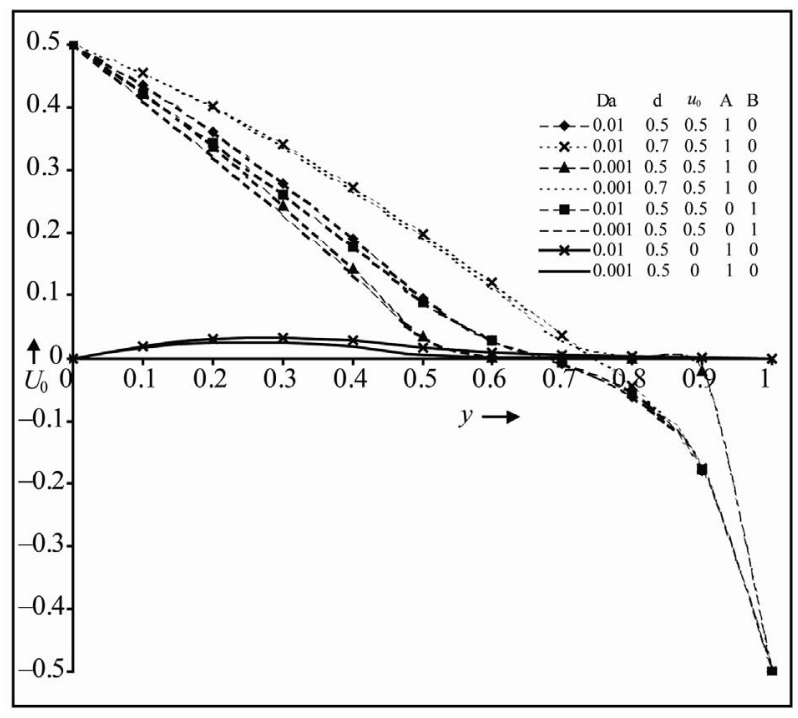

Figure 2. Graph of $U_{0}$ against $y$.

law [19], but for $\mathrm{Da}=10^{-2}$, the velocity does not show the constant nature. It is also observed that the fluid velocity near the heated wall $y=0$ (i.e., $A=1, B=0$ ) is more than the cooled wall $y=0$ (i.e., $A=0, B=1$ ), i.e., heat in the porous medium exerts the restraining force on the fluid.

Figure $\mathbf{3}$ and $\mathbf{4}$ show the effect of viscous and Darcy dissipation terms on the velocity and temperature respectively. The governing equations become non-linear because of existence of these terms whereas very small influence of these terms on both temperature and velocity fields, is observed.

The numerical values of skin-friction on both the plates are also obtained and shown in Tables $\mathbf{1}$ and $\mathbf{2}$ for $u_{0}=0$ and $u_{0}>0$ respectively. $\tau_{1}$ is the skin-friction

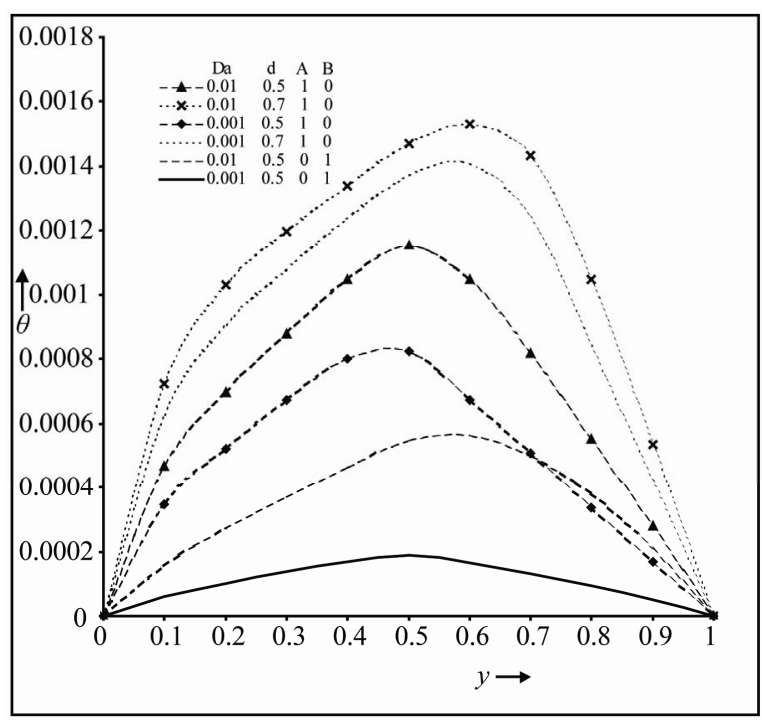

Figure 3. Graph of $\theta$ against $y$.

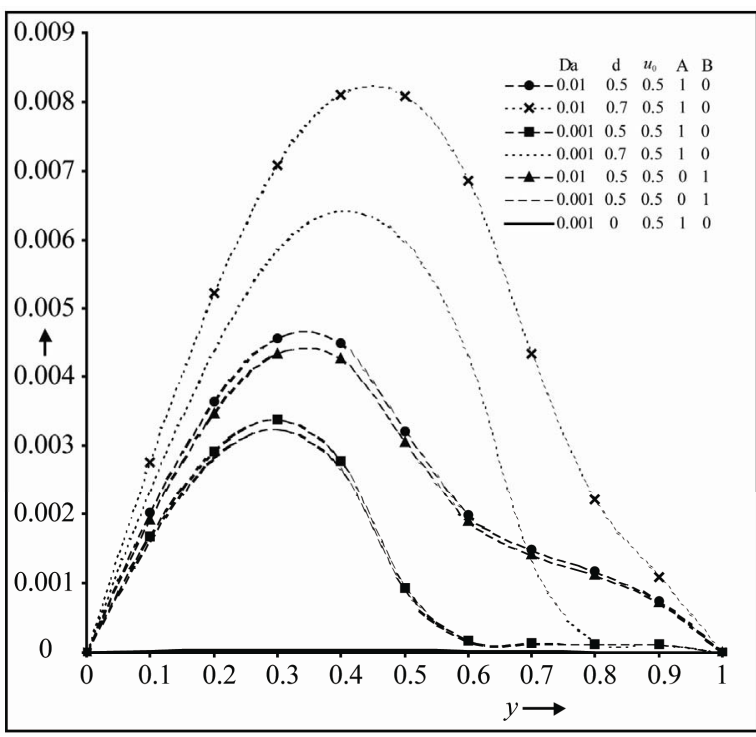

Figure 4. Graph of $U_{1}$ against $y$.

Table 1. Values of skin-friction for $U_{0}=0$.

\begin{tabular}{|c|c|c|c|c|c|c|}
\hline \multirow{2}{*}{$\mathrm{N}$} & \multirow{2}{*}{$\mathrm{Da}$} & \multirow{2}{*}{$d$} & \multicolumn{2}{|c|}{$A=1.0, B=0.0$} & \multicolumn{2}{|c|}{$A=0.0, B=1.0$} \\
\hline & & & 1 & & 1 & \\
\hline \multirow{6}{*}{0} & \multirow{3}{*}{0.1} & 0.3 & 0.2601317 & 0.0772881 & 0.0947285 & 0.2196207 \\
\hline & & 0.5 & 0.2960825 & 0.0916772 & 0.122357 & 0.2310641 \\
\hline & & 0.7 & 0.3221576 & 0.1221194 & 0.1504263 & 0.2647968 \\
\hline & \multirow{3}{*}{0.01} & 0.3 & 0.18 & & & 0.090041 \\
\hline & & 0.5 & 0.2427759 & & & 0.0909297 \\
\hline & & 0.7 & 0.2940734 & 0.0249912 & 0.1107526 & 0.1038333 \\
\hline \multirow{6}{*}{0.1} & \multirow{3}{*}{0.1} & 0.3 & 0.2601631 & & & 0.2196 \\
\hline & & 0.5 & 0.2961261 & 0.0917077 & 0.1223924 & 0.231094 \\
\hline & & 0.7 & 0.3222115 & 0.1221609 & 0.1504735 & 0.2648389 \\
\hline & \multirow{3}{*}{0.01} & 0.3 & 0.1800062 & 0.0101198 & 0.032457 & 0.0900422 \\
\hline & & 0.5 & 0.2427957 & 0.0116499 & 0.065338 & 0.0909317 \\
\hline & & 0.7 & 0.2941141 & 0.025 & 0.1107776 & 0.1038401 \\
\hline
\end{tabular}

Table 2. Values of skin-friction for $\boldsymbol{U}_{\mathbf{0}}=\mathbf{0 . 5}$.

\begin{tabular}{ccccccc}
\hline \multirow{2}{*}{$\mathrm{N}$} & \multirow{2}{*}{ Da } & $d$ & \multicolumn{2}{c}{$A=1.0, B=0.0$} & \multicolumn{2}{c}{$A=0.0, B=1.0$} \\
\cline { 4 - 7 } & & 0.3 & -0.7386463 & 1.836859 & -0.9040495 & 1.9791916 \\
& 0.1 & 0.5 & -0.5860769 & 1.8926462 & -0.7598024 & 2.0320331 \\
& & 0.7 & -0.5739595 & 1.8944114 & -0.7456908 & 2.0370888 \\
0 & & 0.3 & -1.0722803 & 5.0123941 & -1.2198258 & 5.0923165 \\
& 0.01 & 0.5 & -0.6017996 & 5.0225739 & -0.7792456 & 5.1018567 \\
& & 0.7 & -0.3934316 & 5.0685534 & -0.5767524 & 5.1473955 \\
& & & & & & \\
& & 0.3 & -0.7358637 & 1.839478 & -0.9014259 & 1.9816794 \\
& 0.1 & 0.5 & -0.5827951 & 1.8955703 & -0.7567184 & 2.0348034 \\
0.1 & & 0.7 & -0.5700895 & 1.8980549 & -0.7420603 & 2.0405343 \\
& & 0.3 & -1.0708265 & 5.0132408 & -1.2184213 & 5.0931465 \\
& 0.01 & 0.5 & -0.5997182 & 5.023457 & -0.7772541 & 5.1027177 \\
& & 0.7 & -0.3906199 & 5.0697341 & -0.5740707 & 5.148541 \\
\hline
\end{tabular}

when $A=1, B=0$ and $\tau_{2}$ is the skin friction when $A=0, B=1$. Similar effects of Darcy number on skinfriction are observed as mentioned in [16] for $u_{0}=0$, as 
clearly shown in Table 1. Increasing values of skin-friction are observed with increasing width of fluid layer in Table 2. It is also found in Table 2, that increasing Darcy number and dissipation results a very small increment in skin-friciton. The effect of temperature on skin-friction on both the plates is also studied and found that the skin friction on both the plates increases when those are heated.

\section{References}

[1] G. S. Beavers and D. D. Joseph, "Boundary Condition at a Naturally Permeable Wall," The Journal of Fluid Mechanics, Vol. 30, 1967, pp. 197-207. doi:10.1017/S0022112067001375

[2] G. I. Taylor, "A Model for the Boundary Conditions of a Porous Material Part 1,” The Journal of Fluid Mechanics, Vol. 49, No. 2, 1971, pp. 319-326. doi:10.1017/S0022112071002088

[3] S. Richardson, "A Model for the Boundary Conditions of a Porous Material Part 2,” The Journal of Fluid Mechanics, Vol. 49, No. 2, 1971, pp. 327-336. doi:10.1017/S002211207100209X

[4] K. Vafai and S. J. Kim, "Fluid Mechanics of the Interface Region between a Porous Medium and a Fluid Layer: An Alexact Solution," International Journal of Heat Fluid Flow, Vol. 11, No. 3, 1990, pp. 254-256. doi:10.1016/0142-727X(90)90045-D

[5] K. Vafai and S. J. Kim, "On the Limitation of the Brinkman-Forchheimer-Extended Darcy Equation,” International Journal of Heat Fluid Flow, Vol. 16, 1995, pp. 1115. doi:10.1016/0142-727X(94)00002-T

[6] A. V. Kuznetsov, "Thermal Nonequilibrium Forced Convection in Porous Media,” In: D. B. Inghan and I. Pop, Eds., Transport Phenomena in Porous Media, Elsevier, Oxford, 1998, pp. 103-129.

[7] B. Alazmi and K. Vafai, "Analysis of Fluid Flow and Heat Transfer Interfacial Conditions between a Porous Medium and a Fluid Layer," International Journal of Heat Mass Transfer, Vol. 44, No. 9, 2001, pp. 1735-1749. doi:10.1016/S0017-9310(00)00217-9

[8] J. J. Valencia-Lopez and J. A. Ochoa-Tapia, “A Study of
Buoyancy-Driven Flow in a Confined Fluid Overlying a Porous Layer," International Journal of Heat Mass Transfer, Vol. 44, No. 24, 2001, pp. 4725-4736. doi:10.1016/S0017-9310(01)00105-3

[9] D. A. Nield and A. Bejan, "Convection in Porous Media,” New York, Springer-Verlag, 2006.

[10] M. Kaviany, "Principles of Heat Transfer in Porous Media,” Springer-Verlag, New York, 1991.

[11] K. Vafai and C. L. Tien, "Boundary and Inertia Effects on Flow and Heat Transfer in Porous Media," International Journal of Heat Mass Transfer, Vol. 24, No. 2, 1981, pp. 195-203. doi:10.1016/0017-9310(81)90027-2

[12] M. Kaviany, "Laminar Flow through a Porous Channel Bounded by Isothermal Parallel Plates," International Journal of Heat Mass Transfer, Vol. 28, No. 4, 1985, pp. 851-858.

[13] N. Rudriah and S. T. Nagraj, "Natural Convection through Vertical Porous Stratum," International Journal of Engineering Science, Vol. 15, 1977, pp. 589-600. doi:10.1016/0020-7225(77)90055-6

[14] C. Backermann, R. Viskanta and S. Ramadhyani, "Natural Convection in Vertical Enclosures Containing Simultaneously Fluid and Porous Layers,” The Journal of Fluid Mechanics, Vol. 186, 1988, pp. 257-284. doi:10.1017/S0022112088000138

[15] A. Khalili, I. S. Shivakumara and S. P. Suma, "Convective Instability in Superposed Fluid and Porous Layers with Vertical Throughflow," Transactions on Porous Media, Vol. 51, 2003, pp. 1-18. doi:10.1023/A:1021246000885

[16] T. Paul, B. K. Jha and A. K. Singh, "Free-Convection between Vertical Walls Partially Filled with Porous Medium,” Heat and Mass Transfer, Vol. 33, No. 5-6, 1998, pp. 515-519. doi:10.1007/s002310050223

[17] A. K. Singh, T. Paul and G. R. Thorpe, "Natural Convection Due to Heat and Mass Transfer in a Composite System," Heat and Mass Transfer, Vol. 35, 1999, pp. 39-48. doi:10.1007/s002310050296

[18] H. P. G. Darcy, "Les Fontaines Publiques de la villa de Dijon,” Dalmont, Paris, 1856. 